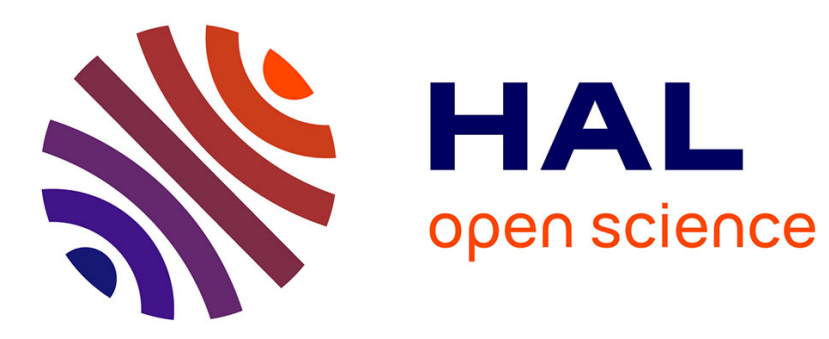

\title{
End-to-End Energy Efficient Geographic Path Discovery With Guaranteed Delivery in Ad Hoc and Sensor Networks
}

\author{
Essia Hamouda Elhafsi, Nathalie Mitton, David Simplot-Ryl
}

\section{- To cite this version:}

Essia Hamouda Elhafsi, Nathalie Mitton, David Simplot-Ryl. End-to-End Energy Efficient Geographic Path Discovery With Guaranteed Delivery in Ad Hoc and Sensor Networks. PIMRC 2008, Sep 2008, Cannes, France. pp.000. hal-00384032

\section{HAL Id: hal-00384032 \\ https://hal.science/hal-00384032}

Submitted on 14 May 2009

HAL is a multi-disciplinary open access archive for the deposit and dissemination of scientific research documents, whether they are published or not. The documents may come from teaching and research institutions in France or abroad, or from public or private research centers.
L'archive ouverte pluridisciplinaire HAL, est destinée au dépôt et à la diffusion de documents scientifiques de niveau recherche, publiés ou non, émanant des établissements d'enseignement et de recherche français ou étrangers, des laboratoires publics ou privés. 


\title{
End-to-End Energy Efficient Geographic Path Discovery With Guaranteed Delivery in Ad Hoc and Sensor Networks
}

\author{
E. H. Elhafsi, N. Mitton, D. Simplot-Ryl \\ IRCICA/LIFL, Univ. Lille 1, CNRS UMR 8022, INRIA Lille-Nord Europe, France \\ essia@cs.ucr.edu, \{nathalie.mitton,david.simplot-ryl\}@inria.fr
}

\begin{abstract}
We propose a novel localized routing protocol for wireless sensor networks (WSN) that is energy-efficient and guarantees delivery. We prove that it is constant factor of the optimum for dense networks. To forward a packet, a node $s$ in graph $G$ computes the cost of the energy weighted shortest path (SP) between $s$ and each of its neighbors which are closer to the destination than itself. It then selects node $x$ which minimizes the ratio of the cost of the SP to the progress towards the destination. It then sends the message to the first node on the SP from $s$ to $x$ : say node $x^{\prime}$. Node $x^{\prime}$ restarts the same greedy routing process until the destination is reached or the routing fails. To recover from the latter scenario, our algorithm invokes Face routing that guarantees delivery. This article is the first to optimize energy consumption of Face routing. First, we build a connected dominating set from graph $G$, second we compute its Gabriel graph to obtain the planar graph $G^{\prime}$. Face routing is applied on $G^{\prime}$ only to decide which edges to follow in the recovery process. On each edge, greedy routing is applied. This two-phase (greedy-Face) End-to-End routing process (EtE) reiterates until the final destination is reached. Simulation results show that EtE outperforms several existing geographical routing on energy consumption metric.
\end{abstract}

\section{INTRODUCTION}

Wireless ad hoc networks, including sensor networks, are receiving a lot of attentions in recent years due to their potential applications in various areas such as monitoring, security and data gathering. However they have some unavoidable limitations compared to fixed infrastructure networks. Energy consumption and scalability are two challenging issues in designing protocols for sensor networks since they operate on limited capacity batteries while the number of deployed sensors could be very large.

In this paper, we focus on designing routing protocols that are scalable, energy efficient and that guarantee delivery in general networks. We consider only localized algorithms where nodes do not need the dissemination of route discovery information nor need to maintain routing tables. Only local information such as the position of the current node holding a packet, the one of its neighbors and the one of the destination are required. Several localized routing protocols [1] with hop count as metric have been proposed to improve scalability. Each node has position information by using a GPS or other localization means [2]; routing decisions are made at each node

This research was partially supported by a grant from CPER Nord-Pasde-Calais/FEDER TAC COMDOM and from the French National Research Agency RNRT SVP (Supervise and Protect). using only local information. Energy-aware localized routing schemes [3], [4], [5] use power consumption as metric. Bust most routing schemes do not guarantee delivery especially in networks with obstacles such as holes and buildings. Several recovery schemes have been proposed [6] to overcome such a drawback. Localized power aware routing algorithms that also guarantee delivery were proposed in [7], [8], [9]. In this work, we propose an end-to-end geographic path discovery protocol $(E t E)$ with the following properties: (i) Localized: In EtE, a node has to be aware only of its location, of its neighbors and of the final destination. (ii) Scalable: EtE is memoryless as no routing information need to be stored at the node. (iii) Loop free: $\mathrm{EtE}$ is loop-free since the greedy step always chooses a node in the forward direction of the destination. (iv) Guaranteed delivery: EtE has 2 routing phases: a localized greedy protocol prone to routing failure and a Face routing step that guarantees delivery invoked when needed. ( $v$ ) Energy efficient: Every routing step EtE takes is energy aware. To avoid expensive long edges, EtE computes an energy weighted localized shortest path (SP) from the relaying node to all its neighbors in the forward direction and selects the one that minimizes the cost of the SP to the progress towards the destination. To avoid expensive short edges, EtE runs Face routing over a connected dominating set (CDS) on which it computes a SP. We prove that the Euclidean length of the path found in greedy phase is within a constant factor of the optimum. For dense uniform networks, we prove that the total energy of a computed path is constant factor of the optimal.

This paper is organized as follows. We introduce the network model and state our assumptions in Section II. Then we briefly cover related work in Section III. In Section IV, we introduce our protocol EtE. We compare EtE performance to existing protocols in Section $\mathrm{V}$ and conclude in Section VI.

\section{PRELIMINARIES}

Network Model While the network model can be arbitrary, the simulations are based on the widely adopted Unit Disk Graph (UDG) model [10]. UDG is defined by $G=(V, E)$, where $V$ represents the set of sensor nodes in the network and there is an edge $e=(u, v) \in E$ between nodes $u$ and $v$ if and only if the Euclidean distance between them $|u v| \leq R$, where $R$ is the transmission radius, equal for all nodes. Let $\mathrm{N}(u)$ be the set of neighbors of node $u$. Let $\mathrm{N}_{x}(u)$ be the 
set of nodes from $\mathrm{N}(u)$ which are closer to $x$ than $u$ that is: $\mathrm{N}_{x}(u)=\mathrm{N}(u) \cap\{\{v\}$ s.t. $|x v|<|x u|\}$. We define the density of the network as the average number of neighbors per node. We assume that each node is aware of its position, the ones of its neighbors and of the destination.

Energy Model The most common energy model [11] is as follows: $\operatorname{power}(r)=r^{\alpha}+c$ if $r \neq 0$, 0 otherwise, where $r$ is the distance separating two neighboring nodes; $c$ is the overhead due to signal processing; $\alpha$ is a real constant $(\geq 2)$ that represents the signal attenuation. The optimal transmission radius, $r^{*}$, that minimizes the total power consumption for a routing task is equal to: $\mathrm{r}^{*}=\sqrt[\alpha]{\frac{\mathrm{c}}{\alpha-1}}$ assuming that nodes can be placed on a line toward the destination [4].

\section{LITERATURE REVIEW}

1) Routing: We briefly describe position based routing schemes relevant to this work. We distinguish between two routing metrics: hop count and power consumption.

Hop count based routing In the greedy method [12], a node holding a packet forwards it to its neighbor $a$ that is the closest to the destination. Though this greedy routing works well in dense networks, it fails if a node $a$ is closer to the destination than any of its neighbors. A routing algorithm that guarantees delivery in 2-D UDG is described in [1]. It applies greedy routing until either the message is delivered or the routing fails. In the latter case, Face routing is applied to recover from failure. Face routing requires the network topology to be a planar graph (i.e., no edges intersect each other). To planarize a graph, several algorithms can be used [1], [13]. Gabriel Graph (GG), for instance, contains edges between nodes $u$ and $v$ iff no other nodes are located inside the circle centered in the middle of edge $(u, v)$ and with diameter $|u v|$. GG has some desirable properties when used for routing in wireless networks such as localized message, free computation and preserving connectivity [1]. GG divides the network into faces. The face that contains the line $(s d)$, where $s$ is the failure node, and $d$ is the destination node, is traversed by right/left-hand rule (placing a virtual hand on the wall of the face) until a node $a$ closer to destination than $s$ is encountered. It has been shown in [6] that Face routing guarantees recovery traversing the first face. Greedy routing continues from $a$ until delivery or another failure node is encountered.

Power consumption based routing Cost over Progress based routing [14], [4] is a localized metric aware greedy routing scheme. A node forwards a packet to the neighbor closer to $d$ such that the ratio of the energy consumed to the progress made (measured as the reduction in distance to $d$ ) is minimized. Though energy efficient, this algorithm does not guarantee delivery. The first article to address guaranteed delivery in power aware localized routing is [7]. It is a GreedyFace-Greedy (GFG) approach where greedy routing is the same as in [4] while Face routing is similar to the one in [1]. One of the drawbacks of Face routing is that it is likely to follow short edges of GG that may be power inefficient.

[9] proposes a GFG energy aware routing with guaranteed delivery. The energy awareness is introduced at the greedy phase where the path to the selected neighbor is enhanced by following the energy weighted SP. The algorithm works as follows. Node $s$ currently holding a packet selects neighbor $a$ closest to $d$ as its temporary destination node. Instead of transmitting directly to $a, s$ computes the energy weighted SP to $a$. This path is followed until a node $b$ closer to $d$ than $s$ is reached. Face routing [1] is applied to recover from failure points. In this work, we will refer to this protocol as SPFSP.

In LEARN [8], a localized energy aware routing is proposed. A node $s$ selects neighbor $b$ inside a restricted neighborhood $(\widehat{b s d} \leq \alpha$ for $\alpha<\pi / 3$ ) that has the largest energy mileage, determined as the ratio $|s b| / \operatorname{power}(|s b|)$. If no such neighbor exists inside the restricted neighborhood, LEARN fails. In the variant LEARN-G, a node switches to greedy routing [12] in case of failure and selects the neighbor closest to the destination. Finally, in the variant LEARN-GFG, a node invokes Face routing when a failure occurs.

Clearly, all existing algorithms use an energy unaware Face routing as a recovery routing scheme. To the best of our knowledge, EtE is the first work to combine Face routing with a power consumption metric.

2) Connected dominating sets: Dominating sets (DS) are defined as follows. Each node in a graph either belongs to a DS or has a neighbor in the DS. Computing the smallest CDS is known to be NP-complete even if the global topology is known. Dai and Wu [15] introduced a generalized DS concept, where coverage can be provided by an arbitrary number of connected 1-hop neighbors. The definition was modified by [5], to avoid message exchanges between neighbors.It is then further simplified in [16] as follows. First, each node $a$ checks if it is an intermediate node. Then if so, node $a$ constructs a subgraph $G^{\prime}$ of its neighbors with higher key values. If $G^{\prime}$ is empty or disconnected then $a$ is in the DS. If $G^{\prime}$ is connected but there exists a neighbor of $a$ which is not a neighbor of any node of $G^{\prime}$ then $a$ is in the CDS. If position information of 1-hop neighbors is available, nodes can decide whether or not they belong to a so defined CDS without exchanging any message with their neighbors.

\section{New Routing ApproACH - EtE Algorithm}

We describe a novel energy efficient georouting $E t E$ with guaranteed delivery. It is based on a GFG routing in which both steps (greedy and Face) are energy aware.

Greedy Routing The greedy step of EtE is based on the SP computation as in [9] but with important differences: first, in the choice of the temporary destination and second, in the computation of the SP. In [9], to send a message, a node $s$

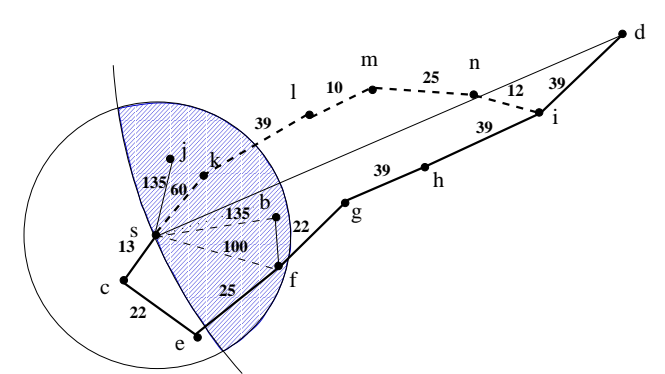

Fig. 1. Greedy routing from node $s$ to $d$. In [9] $s$ selects $b$ and the truncated SP is $s-c-e-f$. EtE algorithm follows a path via node $k$. 
first selects its neighbor closest to the destination (node $b$ in Fig. 1). Then it computes the SP between itself and the selected node. When node $s$ computes the SP towards $b$, it considers all the nodes in its neighborhood (nodes in backward direction $c, e$ and nodes in forward direction $b, f, j, k$ in Fig. 1). In this example, the SP found goes through node $c$ which is not neighbor of $b$ and thus would not be able to compute the SP to $b$. Therefore, the SP needs to be embedded in the message to reach node $b$. In [9] the SP from $s$ to $b$ is truncated at node $f$, first node on the SP closer to $d$ than $s$. In EtE, and contrary to [9], the SP is computed only over nodes in the neighborhood of $s$ in forward direction. In Fig. 1, $s$ considers only nodes in the grey shaded area, nodes $b, f, j$ and $k$. Since each node on the SP that receives the message locally computes the next hop, EtE does not need to embed the SP in the message as in [9] but only the addresses of the source, destination and next hop selected neighbor of $s$. As we show in our simulations, this is a major advantage of EtE over the method in [9] especially when the SP is long. Moreover, since at each step we get closer to the destination, it is clear that EtE is loop-free.

Let $\mathcal{F}(s, d)$ be a function defining the selection criteria of $s$ 's next hop toward $d$. $s$ selects node $b$ which minimizes $\mathcal{F}(s, d)$. Our greedy routing step differs from the one in [9] in the choice of $\mathcal{F}$. In [9], node $s$ selects its neighbor $b$ which is the closest to destination $d$, i. e $\mathcal{F}(s, d)=\min _{u \in \mathrm{N}_{d}(s)}|u d|$.To be more energy efficient, we select this node in a cost-over progress (COP) fashion [17] where we define our cost as the SP cost. Let $x_{0} x_{1} \ldots x_{i} x_{i+1} \ldots x_{n}$, be the nodes on the SP from $s=x_{0}$ to $b=x_{n}$. We define the SP cost as

$$
\operatorname{cost}_{S P}(s, b)=\sum_{i=0}^{n-1} \operatorname{power}\left(\left|x_{i} x_{i+1}\right|\right) \text {. }
$$

Node $s$ selects node $b$ which minimizes $\operatorname{cost}_{S P}(s, b)$ divided by the progress it makes towards destination node $d . \mathcal{F}(s, d)$ is

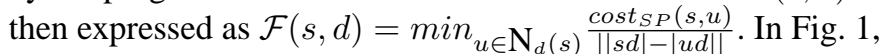
the neighbor selected by the algorithm proposed in [9] is $b$ while EtE selects node $k$. Note that in the given example, the SP from $s$ to $k$ is that link itself, which may happen frequently because of COP optimality criteria for selecting neighbors. In other examples, intermediate nodes may be used on the SP to reduce the overall energy. In such a case, node $s$ sends the message to the first node on the SP towards $b$, which applies the same procedure, formally described in Algorithm 1.

Face Routing Regular Face routing guarantees delivery, but is not energy efficient since it may use too long or too short edges compared to the energy optimal range $r^{*}$. To overcome this drawback, we introduce an energy efficient variant to Face routing. From the original graph $G=(V, E)$ we compute a CDS, $V^{\prime}$ of $V$. We expand $V^{\prime}$ by adding source $s$ and destination $d$ to this set, where $s$ is the node which initiates Face routing step. Let $G^{\prime}=\left(V^{\prime}, E^{\prime}\right) \subset G$ where $E^{\prime} \subset E$ is the set of edges between nodes in $V^{\prime}$. We use the CDS election protocol introduced in [16], however other election protocol may be applied. Since Face routing must be applied on a planarized graph, we generate the $\mathrm{GG}, G^{\prime \prime}=\left(V^{\prime}, E^{\prime \prime}\right)$, from $G^{\prime}$ where $E^{\prime \prime} \subset E^{\prime}$ is the set of edges remaining in the planarized graph. We then run Face routing over $G^{\prime \prime}$. Face

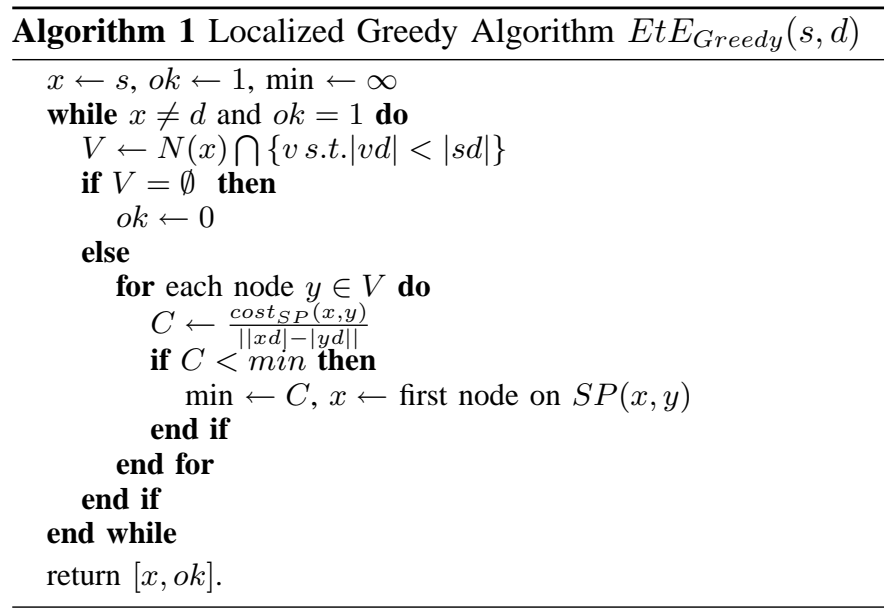

routing guarantees delivery in $G^{\prime \prime}$ since it contains source and destination nodes and preserves connectivity. By considering only edges in the CDS, the routing process avoids short edges. Each node needs to know its neighbors that are in the $\mathrm{CDS}^{1}$. Application of Face routing on $G^{\prime \prime}$ only decides on which edge $(s, b)$ to follow to reach the destination node from a given node $s$ but that edge does not need to be selected since it may be too long $\left(|s b|>r^{*}\right)$. Therefore, we apply the greedy algorithm described in Algorithm 1, where final destination is replaced by temporary destination $b$. That is, $\operatorname{Et} E_{G r e e d y}\left(s^{\prime}, b\right)$ is invoked. If $b$ is closer to the destination node than $s$, node $b$ selects the next hop in the routing path by following the greedy routing described above. Otherwise, it determines the next hop by following Face routing over CDS nodes and computes the $\mathrm{SP}$ to reach it. This process reiterates until the final destination is reached, as formally described in Algorithm 2.

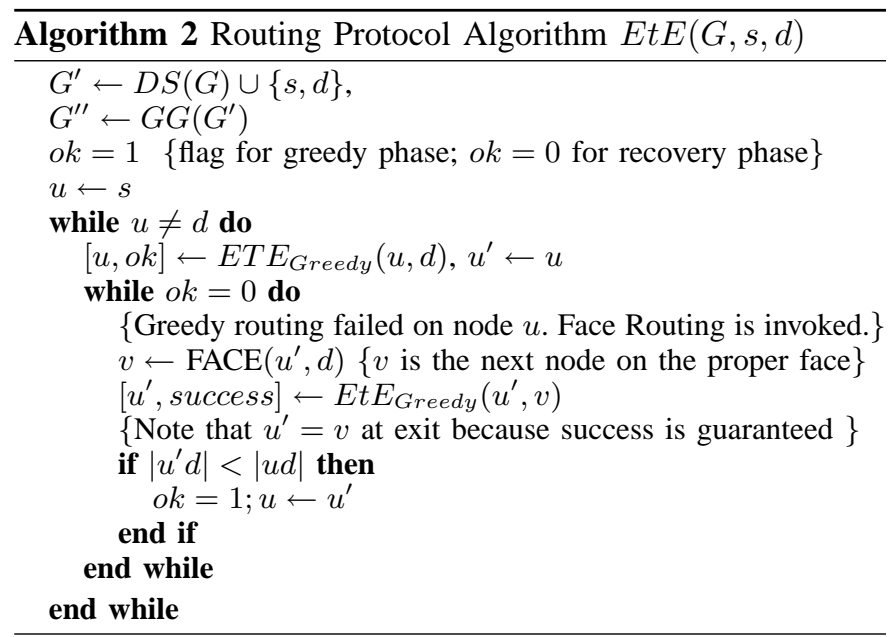

Fig. 2 illustrates a sample execution of EtE algorithm from node 1 to node 8 . Node 1 runs greedy routing and computes the cost of SP towards nodes 2, 19 and 21 (node 23 is not considered since $|23-8|>|1-8|$ ). Node 1 selects node 19 since it provides the lowest COP and sends the packet to node 21 , the first node on the SP towards 19. Node 21 finds

\footnotetext{
${ }^{1}$ This may come from 2-hop position knowledge, or by adding a bit in any message sent by nodes to their neighbors.
} 


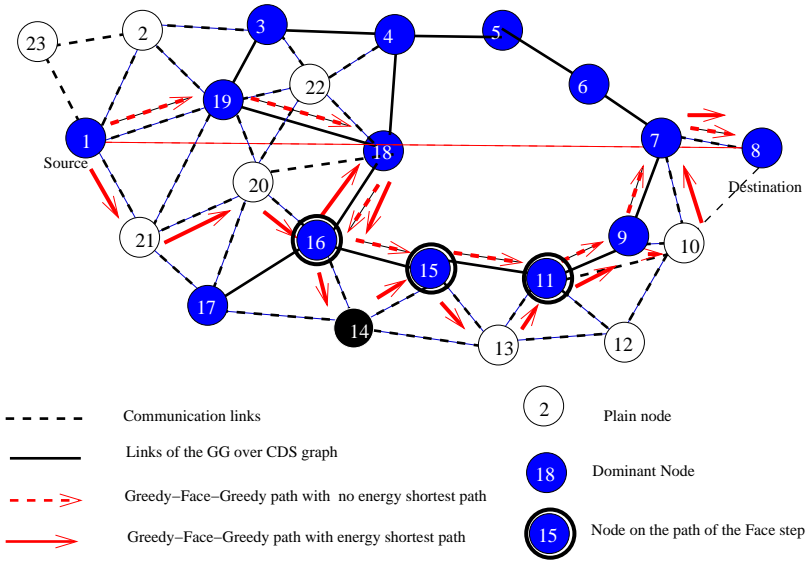

Fig. 2. Illustrative example of EtE algorithm on a sample network.

node 20 as its best forwarder and in this case SP is that link. Node 20 selects node 18 by following a SP through node 16 which then forwards to node 18 where greedy routing fails. Face routing is then invoked to follow edges 18-16 (directly), 16-15 and 15-11 (which are replaced by paths 16-14-15 and 15-13-11 respectively for energy efficiency). Greedy routing then continues till delivery to 11 selecting 10 via 9,9 selecting 7 via 10 , and 10 selecting destination 8 via node 7 .

We now prove more properties of EtE algorithm.

Definition 1: A path meets angular constraints if every hop is within an angle $\theta \leq \alpha<\frac{\pi}{3}, \theta \rightarrow 0$ toward the destination.

Theorem 1: Any path from $s$ to $d$ meeting angular constraint has length that is constant length of the optimum $|s d|$.

Proof: The proof is the same as in [8] where it was restricted specifically to the protocol LEARN. Since that proof does not include any cost function, and is based solely on geometric arguments, it is generally valid for a family of protocols respecting the angular constraint.

Theorem 2: When EtE routing finds a path from source $s$ to destination $d$ in dense graphs, the total energy consumption of the path is within a constant factor of the optimum.

Proof: For any intermediate node $s$ with packet to forward, EtE routing protocol selects the neighbor $u$ such that $\frac{\text { power }_{s, u}}{|s d|-|d u|}$ is minimized. In [8], the neighbor $v$ is selected such that $\frac{\text { power }_{s, v}}{|s v|}$ is minimized. Comparing the COP provided by both choices it then follows that $\frac{\text { power }_{s, u}}{|s d|-|d u|} \leq \frac{\text { power }_{s, v}}{|s d|-|d v|}$. The expected number of hops by both algorithms are $\frac{|s d|}{|s d|-|d u|}$ and $\frac{|s d|}{|s d|-|d v|}$ respectively. The expected energy consumption of the respective paths are then equal to $\left\{\right.$ power $\left._{s, u} \cdot \frac{|s d|}{|s d|-|d u|}\right\}$ and $\left\{\right.$ power $\left._{s, v} \cdot \frac{|s d|}{|s d|-|d v|}\right\}$ which is equivalent to multiplying both sides of the above inequality by $|s d|$. Therefore EtE consumes less energy than LEARN. Since LEARN was proven in [8] to require constant factor of the optimum power for sufficiently dense networks, the theorem follows. Note that the argument is in fact probabilistic with details given in [8].

\section{Performance Evaluation}

We use a home-made $\mathrm{C}$ simulator that assumes no interferences and no packet collisions. The simulated network can be described as follows. Nodes are deployed in a $1000 \times 1000$ square using a Poisson Point Process (node positions are independent) with different node degree ${ }^{2} \delta$. Nodes can adapt their range between 0 and $R=200$. We compare EtE to GFG [6], SPFSP [9] and LEARN-GFG [8] for the same samples of node distribution, same source and destination nodes, both randomly chosen. We evaluate the energy consumption of each algorithm based on the energy model described in Section II. As in [11], we use $c=10^{7}$ and $\alpha=4$, which leads to an optimal range of $r^{*}=100$ [17]. To further evaluate the routing protocols, we computed their energy overhead using as reference the optimal centralized energy weighted SP (Dijkstra algorithm [18]). We let $e_{i}$ and $e^{*}$ be the energy consumed using any described protocol and the centralized SP protocol, respectively. We define the energy overhead as the ratio $\frac{e_{i}-e^{*}}{e^{*}}$. Since one of the novelty of EtE is the use of a CDS, we compare it to its variant EtE' that uses the basic Face phase as in SPFSP. We evaluate the performance of each phase of the protocols independently for better insights on the behavior of the routing schemes. Finally, we evaluate the performance of the routing schemes on a homogeneous network and on a topology with a crescent hole. Because of page restriction, we only present here simulation results for this latter topology.

Paths power consumption Fig. 3 shows that EtE and EtE' outperform existing solutions. They consume only $20 \%$ more energy than the optimal algorithm in a topology with a hole ( $10 \%$ if no hole). The next best performing algorithm is LEARN-GFG, then SPFSP. The worst results are achieved by GFG which consumes $75 \%$ more energy than the optimal solution. Since EtE and EtE' perform the same greedy algorithm, EtE outperforms EtE' only when Face routing is used.

Greedy routing performance Simulations show that greedy routing never rises above $90 \%$ success rate in a topology with a hole (against 100\% hit rate in homogeneous networks when $\delta>15$ ). Fig 4 plots the energy overhead (computed only for successful routings) and shows that our greedy routing algorithm outperforms existing solutions. Moreover, privileging nodes within an angular sector as in LEARN is more efficient than computing a SP toward the node that minimizes the distance to the destination. GFG is the solution which offers the worst performing greedy algorithm.

Face routing performance We evaluate 4 variants of Face routing algorithm independently of the greedy phase: (1) Face used by GFG, run on a GG issued from all nodes in the

\footnotetext{
${ }^{2}$ In such a Poisson Point Process, the total number of nodes is probabilistic and is obtained from a Poisson Law of intensity $\lambda$ with $\lambda=\frac{\delta}{\pi R^{2}}$.
}

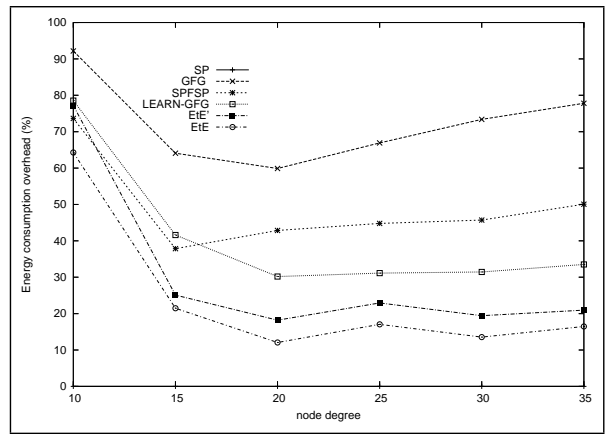

Fig. 3. Energy consumption in a topology with obstacles. 


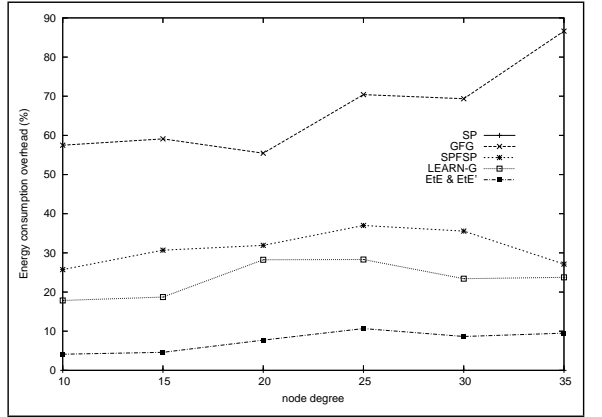

Fig. 4. Greedy energy consumption in a network with obstacles.

network; (2) SPFace (EtE' and SPFSP), which selects the next node using Face and reaches it by performing a SP; (3) DSFace, run over a GG issued from the CDS; and (4) SPDSFace (EtE), which selects the next node using DSFace and reaches it by performing a SP. Results are given in Fig. 5. Notice that SPFace and Face obtain exactly the same results and that by using a CDS, we achieve better energy saving. This can be explained thanks to Fig. 6 which plots face edges average length for the different GGs and the length of the edges followed by the various Face routing algorithms. It shows that, by extracting a GG from the entire set of nodes in the network, we keep only short edges, often smaller than the optimal hop length. So, there is no need to perform a SP. When extracting a GG from a CDS, we keep edges which may easily be longer or equal to the optimal hop length. In this case, performing a SP is of interest. However, this is under condition than the length of faces $l$ is such that $l>r^{*}$, which depends on the maximum radius $R$. For instance, for $\delta=10$, for $150<R<210, l<r^{*}$ if no CDS is used but $l>r^{*}$ with the use of a CDS. Thus, in such cases, using a CDS is of interest, which is supported by Fig. 7 .

\section{CONCLUSION}

We propose a novel routing protocol, EtE, that is both energy efficient and guaranteed delivery. EtE is general enough to be efficiently deployed in a network with arbitrary topology. It is a 2-phase routing protocol that uses an energy optimized greedy routing followed by an energy efficient Face routing scheme. To the best of our knowledge, EtE is the first algorithm that uses an energy efficient Face routing. It is achieved by the integration of the dominating set to optimize the path edge length. Based on our intensive simulations, EtE outperforms various energy-aware routing schemes.

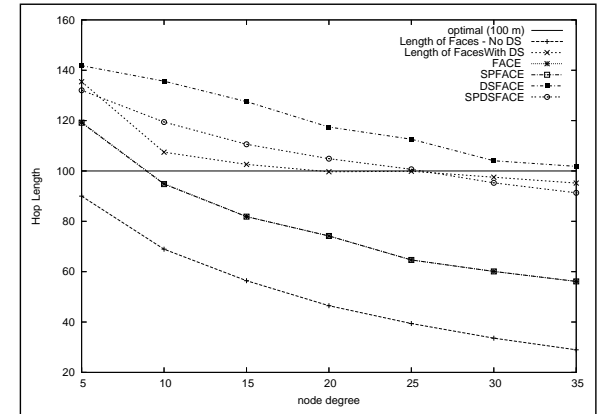

Fig. 6. Edges length for face in both GGs and for the routing paths.

\section{REFERENCES}

[1] P.Bose, P. Morin, I. Stojmenovic, and J. Urrutia, "Routing with guaranteed delivery in ad hoc wireless networks," in DIAL-M, Seattle, WA, USA, 1999, pp. 48-55.

[2] E. H. Elhafsi, N. Mitton, and D. Simplot-Ryl, "Cost over progress based energy efficient routing over virtual coordinates in wireless sensor networks," in IEEE t2pWSN, Helsinki, Finland, 2007.

[3] E. H. Elhafsi and D. Simplot-Ryl, "Flattening the gap between sourcedestination paths in energy efficient greedy georouting in wireless sensor networks," in MSN, Beijing, China, December 2007.

[4] I. Stojmenovic and X. Lin, "Power-aware localized routing in wireless networks," IEEE TPDS, vol. 12, no. 11, pp. 1122-1133, 2001.

[5] D. Simplot-Ryl, I. Stojmenovic, and J. Wu, "Energy efficient backbone construction, broadcasting, and area coverage in sensor networks," Handbook of Sensor Networks, Wiley, pp. 343-379, 2005.

[6] H. Frey and I. Stojmenovic, "On delivery guarantees of face and combined greedy-face routing in ad hoc and sensor networks," in MOBICOM, Los Angeles, CA, USA, 2006.

[7] I. Stojmenovic and S. Datta, "Power and cost aware localized routing with guaranteed delivery in wireless networks," Wireless Comm. and Mobile Compt., vol. 4, no. 2, pp. 175-188, 2004.

[8] Y. Wang, W.-Z. Song, W. Wang, X.-Y. Li, and T. Dahlberg, "LEARN: Localized energy aware restricted neighborhood routing for ad hoc networks," Sensor and Ad Hoc Comm. and Networks, vol. 2, 2006.

[9] J. A. Sanchez and P. M. Ruiz, "Exploiting local knowledge to enhance energy-efficient geographic routing," in MSN, Hong Kong, China, 2006.

[10] B. Clark, C. Colbourn, and D. Johnson, "Unit disk graphs," DISCMATH, vol. 86, no. $1-3$, pp. 165-177, 1990.

[11] V. Rodoplu and T. Meng, "Minimizing energy mobile wireless networks." IEEE Journal on Selected Areas, vol. 17, pp. 1333-1347, 1999.

[12] G. Finn, "Routing and addressing problems in large metropolitan-scale," Internetworks, ISI Research Report ISU/RR-87-180, March 1987.

[13] G. Toussaint, "The relative neighborhood graph of a finite planar set," Pattern Recognition, vol. 12, no. 4, pp. 261-268, 1980.

[14] I. Stojmenovic, "Localized network layer protocols in sensor networks based on optimizing cost over progress ratio," IEEE Network, vol. 20, pp. 21-27, January/February 2006.

[15] F. Dai and J. Wu, "An extended localized algorithm for connected dominating set formation in ad hoc networks," IEEE TPDS, 2004.

[16] J. Carle and D. Simplot-Ryl, "Energy efficient area monitoring by sensor networks," IEEE Computer Magazine, vol. 37, pp. 40-46, 2004.

[17] J. Kuruvila, A. Nayak, and I. Stojmenovic, "Progress and location based localized power aware routing for sensor networks," IJDSN, vol. 2, 2006.

[18] E. Dijkstra, "Solution of a problem in concurrent programming control," Communications of the ACM, vol. 8, no. 9, September 1965.

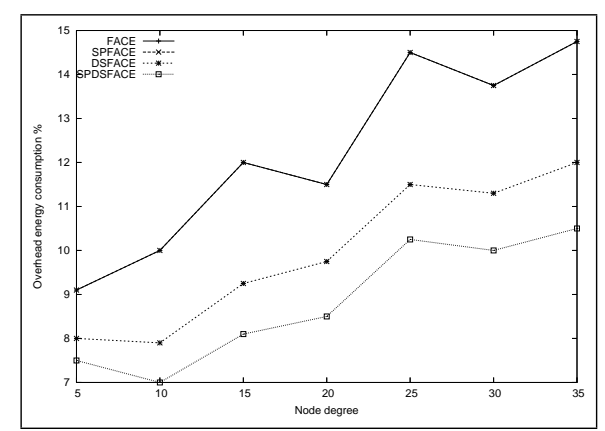

Fig. 5. Face routing features of the different routing protocols.

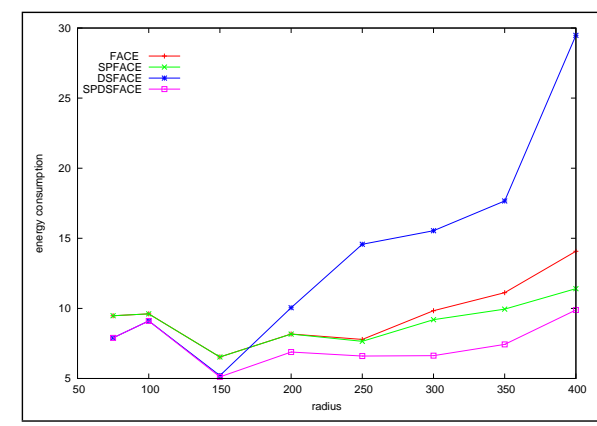

Fig. 7. Energy consumption as a function of $R$ for $\delta=10$. 